\title{
The Effects of Influenza Vaccination on Immune Function in Patients with Chronic Fatigue Syndrome/Myalgic Encephalomyelitis
}

\author{
Ekua Weba Brenu ${ }^{1,2^{*}}$, Mieke van Driel ${ }^{1,3}$, Donald R. Staines ${ }^{1,4}$, Sanne Kreijkamp-Kaspers ${ }^{1,2}$, \\ Sharni Lee Hardcastle ${ }^{1,2}$, Sonya Maree Marshall-Gradisnik ${ }^{1,2}$ \\ ${ }^{1}$ School of Medical Science, Griffith Health Institute, Griffith University, Gold Coast Campus, Gold Coast, Australia; ${ }^{2}$ The National \\ Center for Neuroimmunology and Emerging Diseases, Griffith University, Gold Coast, Australia; ${ }^{3}$ Discipline of General Practice, \\ School of Medicine, University of Queensland, Brisbane, Australia; ${ }^{4}$ Queensland Health, Gold Coast Public Health Unit, Gold Coast, \\ Australia. \\ Email: "e.brenu@griffith.edu.au
}

Received August 13 ${ }^{\text {th }}, 2012$; revised September $14^{\text {th }}, 2012$; accepted October $10^{\text {th }}, 2012$

\begin{abstract}
Immune dysfunction is a hallmark of Chronic Fatigue Syndrome/Myalgic Encephalomyelitis (CFS/ME). The purpose of this pilot study was to identify the effects of influenza vaccination on immune function in patients with CFS/ME. We included 7 patients meeting the Centre for Disease Control and Prevention criteria (CDC 1994) for ME/CFS and 8 control subjects. Bloods were collected from all participants prior to vaccination with Influvac, a trivalent inactivated influenza vaccine (TIV), 14 and 28 days following vaccination. The immune parameters examined include Natural Killer (NK) phenotypes, NK cytotoxic activity, FOXP3 and Th1/Th2/Th17 related cytokines. Flow cytometric protocols were employed. There was no significant difference in NK phenotypes and Tregs numbers between CFS/ME patients and healthy controls. However, NK activity was significantly decreased at baseline and at 28 days, while at 14 days it significantly increased in the CFS/ME patients compared to the healthy controls. Th1 pro-inflammatory cytokines increased considerably in the CFS/ME patients at 28 days compared to the non-fatigued controls. Only one Th2 cytokine, IL-4, increased in the CFS/ME participants. FOXP3 expressing Tregs only increased significantly at day 28 post vaccination in the CFS/ME patients compared to the healthy controls. Self-rated wellbeing was lower for patients at day 28 while at baseline and day 14 no differences were observed. In this pilot study immunization with influenza vaccine is accompanied by a degree of immune dysregulation in CFS/ME patients compared with controls. While vaccination may protect CFS/ME patients against influenza, it has the ability to increase cytotoxic activity and pro-inflammatory reactions post vaccination. The role of Tregs in promoting a toxic effect at 28 days post-vaccination in our patient group cannot be ruled out. The benefits of influenza vaccine still likely outweigh the risks CFS/ME patients experience following vaccination.
\end{abstract}

Keywords: Chronic Fatigue Syndrome; Influenza; Vaccination; Natural Killer Cells; Cytokines

\section{Introduction}

The influenza virus is genetically variable thus new vaccines are developed annually to ensure effective protecttion against new strains of the virus. Variation in the virus mainly occurs in the main viral glycoproteins, haemaglutinin $(\mathrm{H})$ and neuraminidase $(\mathrm{N})$ [1]. The immune system is important in eliminating and clearing viral infections. Usually, during an influenza infection, recognition of an infected cell occurs via the retinoic acid inducible gene I (RIG-I) while influenza genomic RNA is detected by the Toll-like receptor 7 (TLR-7) [2-4].

\footnotetext{
"Corresponding author.
}

This stimulates the generation and production of soluble proteins belonging to the type I interferon (IFNs) family, which in turn suppress viral reproduction [5]. Both the innate and adaptive immune systems are required to combat viral infections. In the innate immune system, macrophages secrete IL-1 $\beta$ and IL-18 [6] while adaptive immune system cells such as $\mathrm{CD} 4^{+} \mathrm{T}$ and $\mathrm{CD} 8^{+} \mathrm{T}$ cells generate influenza specific memory cells. These cells are equipped to recognize conserved sequences within the virus that serve as a protective mechanism for the virus by making them undetectable for the vaccine $[7,8]$.

Chronic Fatigue Syndrome/Myalgic Encephalomyelitis (CFS/ME) is a disease with unknown aetiology 
characterized by severe fatigue and flu-like symptoms $[9,10]$. CFS/ME affects a substantial number or people worldwide and has been associated with a number of viruses [11-15]. Although the exact cause of CFS/ME is unknown, serological studies have shown high rates of viral titres in CFS/ME patients [15-18]. This may indicate an inability to effectively clear infections as immune function has been shown to be decreased in CFS/ME patients when compared to non-CFS/ME individuals [19-22]. A number of factors such as mental, physical, psychological stresses and viral infections may exacerbate symptoms of CFS/ME [23].

Administration of TIV to CFS/ME patients in general, is a controversial issue [1,24-29]. This is mainly related to the view that vaccines may affect the immune system in CFS/ME patients resulting in a worsening of symptoms and consequently physical health. Incidentally, a survey of CFS/ME patients revealed that $31 \%$ of patients who had not received influenza vaccination were of the view that immunization was hazardous to their health. However, among patients who had been vaccinated and developed complications $28 \%$ were optimistic and $26 \%$ agreed that vaccines were not detrimental to their health [29]. The degree of support for the influenza vaccine was higher among CFS/ME patients who had experienced positive outcomes from previous vaccinations; $45 \%$ rated vaccinations as favourable, while $55 \%$ agreed it was advantageous [29]. A recent study investigated the effect of influenza vaccination on immune response and noted that in some cases, administration of whole virus influenza vaccine was observed to raise antibody titres, with no significant side effects in the CFS/ME group in comparison to the non-CFS/ME group [27]. Additionally, there was no significant difference in the symptoms of CFS/ME patients with or without the influenza vaccine 6 weeks after vaccination [27]. The results of this first study suggest there is no demonstrable impact on the ability to generate antibodies. However, the effect on cellular immunity, which is known to be affected in CFS/ME patients, has not been studied before. Whole virus influenza vaccines are also inactivated however due to the adverse events of whole virus influenza vaccines, only split virus influenza vaccines have been available in the US since 2001.

The purpose of this study is to explore the effects of influenza vaccination on immune function in CFS/ME patients, by examining and comparing NK activity, NK phenotypes, cytokine secretion pre, 14 and 28 days post vaccination.

\section{Materials and Methods}

\subsection{Participants Selection}

A total of 15 participants were selected from a database of patients living in the South East Queensland region of Australia [30]. Seven patients were classified as having CFS/ME using the Centre of Disease Prevention and Control (CDC) 1994 case definition [31]. The nonCFS/ME controls comprised of 8 participants from the same region. Written consent was obtained from all participants prior to blood sampling. A volume of $40 \mathrm{~mL}$ of non-fasting morning blood samples were collected from the antecubital vein into lithium heparin and EDTA tubes. Full blood count measurements were obtained prior to immunological assessments.

\subsection{Vaccine Intervention}

Prior to vaccination contraindications for vaccination were checked as per the Australian immunisation handbook [32]. In the absence of contraindications the vaccine was administered by intramuscular injection in the deltoid muscle by a doctor. The patient was observed for at least 15 minutes post vaccination to monitor any immediate adverse effects. The type of influenza vaccine used was Influvac (Solvay Pharmaceuticals) an inactivated influenza vaccine. Each $0.5 \mathrm{~mL}$ pre-filled syringe contains $15 \mu \mathrm{g}$ haemagglutinin of each of the three recommended strains produced for the 2011 flu season (2011) A/California/, 7/2009 (H1N1) - like strain, A/Perth/, 16/2009 (H3N2) - like strain and B/, Brisbane/60/2008like strain). The vaccine batch number was documented on the day of administration. At follow up patients were asked to report any adverse effects following vaccination. At every blood collection participants self-rated their general wellbeing, cold and flu symptoms and mood on a visual analogue scale (VAS), with the best imaginable or no symptoms rated as 0 and the worst imaginable as 10 .

\subsection{NK Cytotoxic Activity}

A flow cytometric based cytotoxic protocol was used to measure NK cytotoxic activity $[19,30,33]$. This involved isolating peripheral blood mononuclear cells (PBMCs) from whole blood samples using density gradient centrifugation. Subsequently cells were labeled with $0.4 \%$ PKH-26 (Sigma, St. Louis, MO). PBMCs were then incubated with $\mathrm{K} 562$ cells, for 4 hours at $37^{\circ} \mathrm{C}$ in $95 \%$ air, $5 \% \mathrm{CO}_{2}$ using a ratio of 25 (NK cells): 1 (K562). Apoptosis of K562 cells were analyzed [19,30] via FACSCalibur flow cytometry (BD Bioscience, San Jose, CA), after labeling cells with Annexin V-FITC and 7-AAD reagent (BD Pharmingen, San Diego, CA).

\subsection{NK Phenotypes Quantitation}

NK cell subsets distribution, i.e. CD56brightCD16 ${ }^{-}$NK cells and CD56dimCD16 ${ }^{+}$NK cells was determined as previously described $[19,30]$. Preferential isolation of 
NK cells was performed using a negative selection system, RosetteSep Human Natural Killer Cell Enrichment Cocktail (StemCell Technologies, Vancouver, BC) from whole blood. NK cells were labelled with monoclonal antibodies for CD56 (FITC) and CD16 (PE) (BD Pharmingen, San Jose, CA).

\subsection{Cytokine Secretion Following Mitogenic Stimulation}

Cytokines were measured following mitogenic stimulation of cultured PBMCs. In brief PBMCs were isolated from whole blood using ficoll-hypaque density gradient centrifugation. PBMCs were placed in cell culture media with or without $1 \mu \mathrm{g}$ of phytohemagluttinin for 72 hours at a concentration of $1 \times 10^{6}$ cells $/ \mathrm{mL}$ in RPMI culture media at $37^{\circ} \mathrm{C}$ in $95 \%$ air with $5 \% \mathrm{CO}_{2}$. Cell supernatants were collected immediately and stored at $-80^{\circ} \mathrm{C}$ for later assessment. Th1, Th2 and Th17 cytokine profiles were determined using the cytometric bead array kit (BD Pharmingen, San Jose, CA) $[30,34]$. The concentrations of the following cytokines IL-2, IL-4, IL-6, IL-10, tumor necrosis factor (TNF)- $\alpha$, interferon (INF)- $\gamma$ and IL-17A were measured.

\subsection{Examination of Regulatory $T$ Cells in CFS/ME Patients.}

Assessment of Tregs was performed as previously described [30]. PBMCs isolated from $10 \mathrm{ml}$ of whole blood were washed and stained with monoclonal antibodies FITC-CD4 and APC-CD25 (BD Pharmingen, San Diego, CA). Cells were then permeablised and fixed with buffers containing diethylene glycol and formaldehyde. Subsequently, cells were stained with PE-FOXP3 and evaluated on the flow cytometer. FOXP3 was determined on $\mathrm{CD} 4^{+} \mathrm{CD} 25^{+} \mathrm{T}$ cells.

\subsection{Statistical Analysis}

Statistical analysis was performed using SPSS software version 18.0. All data are reported as means plus/minus standard error of the mean $( \pm \mathrm{SEM})$. Analysis of variance test (ANOVA) and repeated measures was used to compare continuous data. As the different variables were examined at three different time points, time was considered as the within-subjects factor while group was the between-subject factor. Changes in data between time points were further assessed using Bonferroni post-hoc analysis. To assess within subject stability Pearson and Spearman's rank correlations were determined between the three time points. Significance was set at $p$-values less than or equal to 0.05 .

Approval for this study was obtained from the Bond University Human Research Ethics Committee.

\section{Results}

\subsection{Participants Information}

The study population consisted of $7 \mathrm{CFS} / \mathrm{ME}$ patients with an average age of 48 years old and 8 controls all females with an average age of 38 years old. All patients fulfilled the CDC criteria for CFS. At baseline there were no statistically significant differences between the two groups but at day 14 and particularly day 28 post vaccinetion, the CFS/ME patients recorded a significantly lower general wellbeing compared to the controls (Figure 1).

\subsection{Assessment of NK Cytotoxic Activity}

NK cytotoxic activity was significantly decreased $(p<$ 0.05 ) in CFS/ME patients compared to the control group prior to vaccination and at 28 days following vaccination. However, there was no significant decrease in the two groups at 14 days post vaccination (Figure 2).

\subsection{Distribution of NK Cells}

NK phenotypes were classified as CD56dimCD16 ${ }^{+}$and CD56brightCD16 ${ }^{-} \mathrm{NK}$ cells. CD56dimCD16 ${ }^{+}$and CD56brightCD16 ${ }^{-}$NK cells numbers were not significantly different at any time point in CFS/ME patients compared to non-fatigued controls.

\subsection{Cytokine Secretion Pre and Post Vaccination}

Cytokine secretion following mitogenic stimulation did not differ before and 14 days after vaccination in the $\mathrm{CFS} / \mathrm{ME}$ and control groups. At 28 days post vaccination, significant increases in IL-4, IL-17A, IFN- $\gamma$ and TNF- $\alpha$ were observed in the CFS/ME patient compared to the

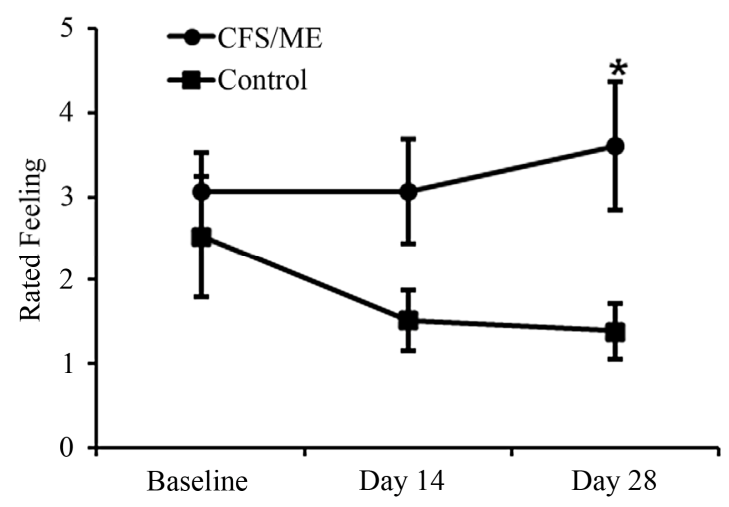

Figure 1. Self rated wellbeing in the CFS/ME and non-fatigued controls. The graph represents wellbeing of the participants (CFS/ME and non-fatigued controls) throughout the study that is at baseline, day 14 and day 28 . The $a$ represent control data while $\bullet$ depicts the CFS/ME data. Data are presented as means \pm SEM. "Represents a statistical significant result in relation to controls, $p<0.05$ vs nonfatigued control. 


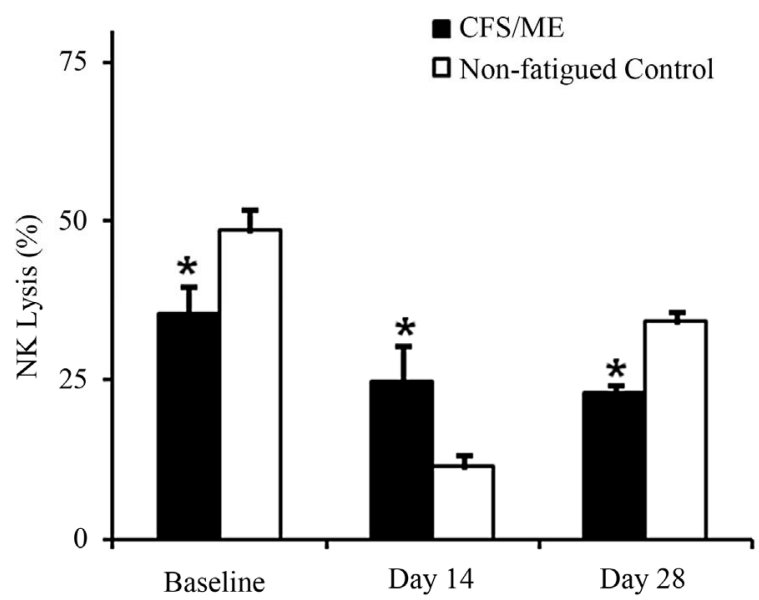

Figure 2. NK cytotoxic activity pre and post vaccination. NK cytotoxic activity is presented as the \% of $\mathrm{K562}$ cells lysed by NK cells pre (baseline) and post vaccination (day 14 and day 28) in the non-fatigue control group (white bars) while and the CFS/ME group (black bars). "Represents a statistical significant result in relation to controls. Data are presented as means \pm SEM. controls (Figures 3(a)-(d). There was no significant difference in FOXP3 pre and 14 days post vaccination. However, an increase in FOXP3 was observed 28 days post vaccination in the CFS/ME group compared to the controls (Figure 4).

\section{Discussion}

Our pilot study suggests that vaccination with seasonal Influenza vaccine, Influvac may affect cellular immune function in $\mathrm{CFS} / \mathrm{ME}$ patients, such as increase of NK function and differential changes in the expression of release of certain proteins by lymphocytes. However, the phenotypes of immune cells such as NK phenotypes may not necessarily be affected by influenza vaccines as these cells remained unchanged post vaccination. An increase in $\mathrm{T}$ cell related proteins occurred post vaccination. Overall wellbeing of CFS/ME patients was also significantly lower on the last day of the study. The idea behind the seasonal administration of the influenza vaccine relates to its ability to protect against the effects of

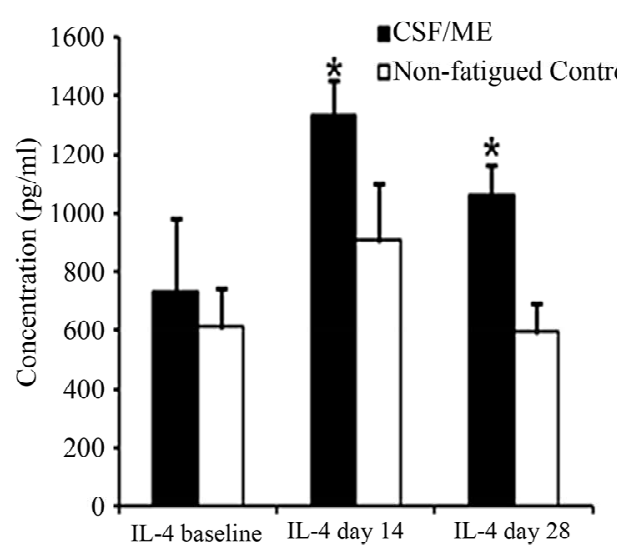

(a)

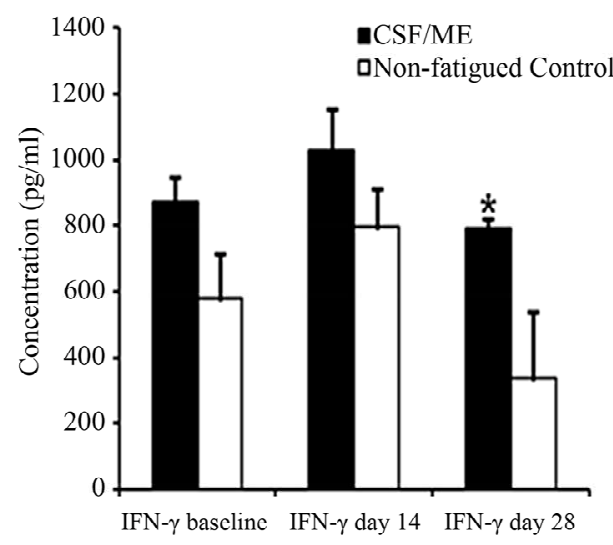

(c)

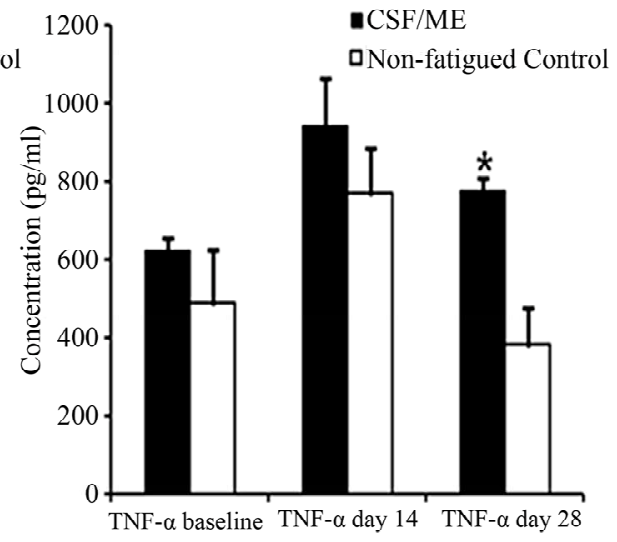

(b)

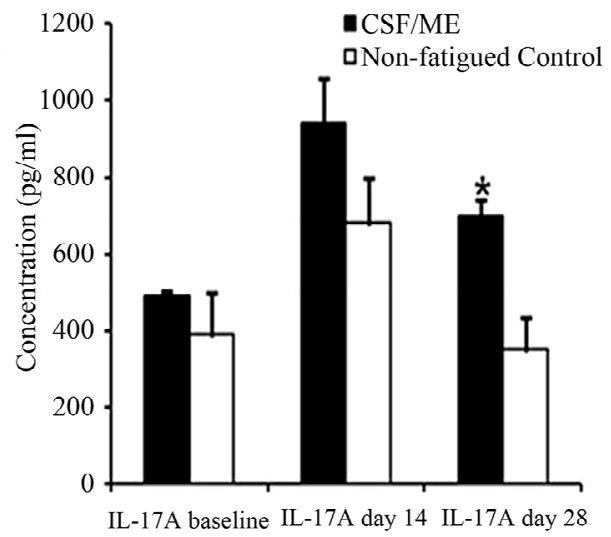

(d)

Figure 3. Levels of different cytokines pre and post vaccination. The graph (a) represents the secretion of IL-4; (b) TNF- $\alpha$; (c) IFN- $\gamma$ and (d) IL-17A pre (baseline) and post vaccination (day 14 and day 28). The white bars depict data from the non-fatigued controls and the black bars are representative of the CFS/ME patients. Data are presented as means \pm SEM. "Represents a statistical significant result in relation to controls, $p<0.05$. 


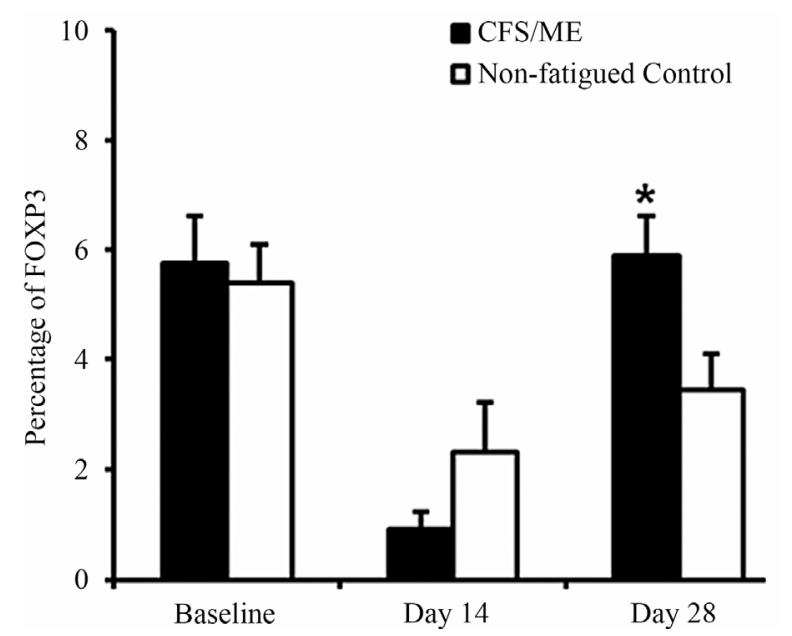

Figure 4. Intracellular FOXP3 expression pre and post vaccination. The graph represents the FOXP3 production from intracellular staining of Treg cells pre (baseline) and post (day 14 and day 28) vaccination. The white bars depict data from the non-fatigued controls and the black bars are representative of the CFS/ME patients. Data are presented as means \pm SEM. " Represents a statistical significant result in relation to controls, $p<0.05$.

different strains of the influenza virus. Previous studies have confirmed the protective effects of certain vaccines on the immune system in CFS/ME patients $[1,26,28]$. To our knowledge this is the first study to report the impact of influenza vaccination on the cellular immune system in CFS/ME patients.

Immune cell function, in particular, NK cytotoxic activity was decreased prior to vaccination and 28 days post vaccination, and increased at day 14 in the CFS/ME group compared to the non-fatigued controls. This suggests that TIV affected responses in the immune system 14 days after vaccination, however, this immune response returned to its initial status at 28 days following vaccination as NK cytotoxic activity decreased at both baseline and day 28. The cause of the increase in NK activity in the CFS/ME patients following vaccination is not explicitly known, however, administration of TIV and LAIV influenza vaccines have the potential to induce cytotoxic activity in NK cells. Importantly, these vaccines are known to generate memory cytotoxic cells in cytotoxic $\mathrm{CD}^{+} \mathrm{T}$ cells that are important for preventing influenza $[35,36]$.

The mechanism of cytotoxic activity involves the activation of granule dependent pathways, which facilitate the release of cytolytic proteins, perforin and granzymes, into the cytoplasm of the viral or tumor cell. Influenza virus decreases the expression of these molecules, specially perforin [37]. Administration of TIV increases the expression of perforin in $\mathrm{CD}^{+} \mathrm{T}$ cells and this likely occurs in NK cells, thereby enhancing cytotoxic activity in some cells [38]. In adults, reduced levels of granzyme $B$ increase the threat of influenza. However, higher levels of granzyme B and NK activity have been observed in older compared to younger individuals following influenza vaccination $[39,40]$. This presupposes that the influenza vaccine may enhance cytotoxic activity by prolonging the cytotoxic process and increasing the presence of lytic proteins in cytotoxic cells. Accordingly, the type of influenza vaccine used may have an impact on the activity of NK cells. For example, NK cell activity is prolonged in the presence of TIV [41]. Hence, this may be a contributory factor to the elevated cytotoxic activity following influenza vaccination with the TIV used in our patients. These findings may support the rationale that influenza vaccines are protective rather than harmful in most individuals. Additionally, the significant decrease in cytotoxic activity may be correlated with the low levels of wellbeing 28 days after vaccination. This is consistent with the literature that reduced well-being with low immune function, particularly decreases in NK cell cytotoxic activity [42]. However, the rise in inflammatory cytokines at this time point, together with potentially pathogenic regulatory $T$ cell activity may suggest enhanced toxicity contributing to a lowered sense of wellbeing.

The majority of the cytokines that were significantly increased in the CFS/ME patients were Th1 cytokines including IFN- $\gamma$ and TNF- $\alpha$, IL-17 was also increased. IL-4 was the only Th2 cytokine that was increased in the $\mathrm{CFS} / \mathrm{ME}$ patients compared to the non-fatigued controls. Hence, it can be deduced that influenza vaccines may increase the production of both pro- and anti-inflammatory cytokines in the CFS/ME patients. This is consistent with the observation that CFS/ME patients have some background dysregulation of their immune system and correlates with the clinically reported worsening of symptoms. However Influenza vaccines can cause headaches, arthralgia, fever, nausea and malaise [43], which coincidentally are symptoms of CFS/ME.

The effect of the influenza vaccine on the immune system, in terms of cytokine response is not immediate and seems to occur at a later stage. In the adaptive immune system, vaccines such as the TIV effectively prompt Th1 related immune response which is characterized by the presence of IL-2 [44]. Similarly, an increase in IL-17 was observed after vaccination of mice with TIV. Although these studies were done in mice, they may provide a snapshot of the actual effects of inactivated influenza vaccines on the human immune system. Cytokine effects were only observed 28 days post vaccination and prior to this, no significant changes in cytokines were observed. A predominant Th1 immune response suggests that an increase or exacerbation in 
pro-inflammatory activity possibly occurs in CFS/ME patients. However, in a previous study, we reported that cytokines tend to fluctuate in CFS/ME patients and this may persist following vaccination, although, the samples size was too small to draw further conclusions. Similarly, in vitro examination of different types of influenza vaccines shows the ability to stimulate a Th1 immune type response in PBMCs, which again was more prevalent in the older compared to the younger participants $[45,46]$. This is suggestive of an improvement in immune response as a consequence of the administration of vaccines.

This pilot study demonstrates the potential effects of TIV on immune function in CFS/ME patients compared to non-fatigued individuals. The cause of the heightened cytotoxic activity is not clear, however, this increase in cytotoxic function is subsequently followed by an increase in pro-inflammatory cytokines. Whether this substantial increase in cytokines is related to the influenza vaccine is not entirely known, as cytokines have the tendency to oscillate; however, no oscillations were observed pre vaccination and at day 14. Interestingly, altered Treg function at 28 days may also influence lowered wellbeing in this patient group. More investigations are required to see if the Treg subset may be pathogenic. The influenza vaccine may play a role in modifying the pattern of immune expression observed in our CFS/ME patients.

Our study has limitations. A relatively small sample size of patients and controls was used and this may have biased the changes observed in the parameters measured. Additionally, immune measurements were not taken within the first few days post vaccination. These measurements may be important in determining the rate of change in cytotoxic activity and cytokines immediately after administering the vaccine. In addition, only one type of vaccine, i.e. the TIV, was investigated. Perhaps future studies on the effects of other vaccines on the immune system may be important in disseminating the type of vaccines to recommend to $\mathrm{CFS} / \mathrm{ME}$ individuals.

\section{Conclusion}

In conclusion, the challenge of determining the most effective intervention against influenza in individuals with complex disorders such as CFS/ME remains to be determined. Influenza vaccines such as TIV may be important in increasing immunity against pathogens by increasing cytotoxic mechanisms and pro-inflammatory cytokines. However, as the effectiveness of influenza vaccines is not $100 \%$ in all individuals, it is possible that in some cases vaccination against influenza may be protective as it has the ability to increase cytotoxic activity and proinflammatory reactions post vaccination, while adverse effects may ensue in others. These vaccines should be administered with caution in patients with complex disorders where immune function is severely compromised. The advantage of influenza vaccine however, may likely offset the risks associated with CFS/ME.

\section{REFERENCES}

[1] P. Magnus, O. Brubakk, H. Nyland, B. H. Wold, H. K. Gjessing, I. Brandt, T. Eidem, H. Nokleby and G. SteneLarsen, "Vaccination as Teenagers against Menin-Gococcal Disease and the Risk of the Chronic Fatigue Syndrome," Vaccine, Vol. 27, No. 1, 2009, pp. 23-27. doi:10.1016/j.vaccine.2008.10.043

[2] S. S. Diebold, T. Kaisho, H. Hemmi, S. Akira and C. Reis e Sousa, "Innate Antiviral Responses by Means of TLR7Mediated Recognition of Single-Stranded RNA," Science, Vol. 303, No. 5663, 2004, pp. 1529-1531. doi:10.1126/science.1093616

[3] H. Kato, S. Sato, M. Yoneyama, M. Yamamoto, S. Uematsu, K. Matsui, T. Tsujimura, K. Takeda, T. Fujita, O. Takeuchi and S. Akira, "Cell Type-Specific Involvement of RIG-I in Antiviral Response," Immunity, Vol. 23, No. 1, 2005, pp. 19-28.

[4] J. M. Lund, L. Alexopoulou, A. Sato, M. Karow, N. C. Adams, N. W. Gale, A. Iwasaki and R. A. Flavell, "Recognition of Single-Stranded RNA Viruses by Toll-Like Receptor 7," Proceedings of National Academy of Sciences of the United States of America, Vol. 101, No. 15, 2004, pp. 5598-5603.

[5] A. Garcia-Sastre and C. A. Biron, "Type 1 Interferons and the Virus-Host Relationship: A Lesson in Detente," Science, Vol. 312, No. 5775, 2006, pp. 879-882. doi:10.1126/science. 1125676

[6] J. Pirhonen, T. Sareneva, M. Kurimoto, I. Julkunen and S. Matikainen, "Virus Infection Activates IL-1 Beta and IL-18 Production in Human Macrophages by a Caspase-1-Dependent Pathway," Journal of Immunology, Vol. 162, No. 12, 1999, pp. 7322-7329.

[7] G. T. Belz, S. Bedoui, F. Kupresanin, F. R. Carbone and W. R. Heath, "Minimal Activation of Memory $\mathrm{CD} 8^{+} \mathrm{T}$ Cell by Tissue-Derived Dendritic Cells Favors the Stimulation of Naive CD ${ }^{+}$T Cells," Nature Immunology, Vol. 8, 2007, pp. 1060-1066. doi:10.1038/ni1505

[8] J. F. Fonteneau, M. Gilliet, M. Larsson, I. Dasilva, C. Munz, Y. J. Liu and N. Bhardwaj, "Activation of Influenza Virus-Specific $\mathrm{CD}^{+}$and $\mathrm{CD}^{+} \mathrm{T}$ Cells: A New Role for Plasmacytoid Dendritic Cells in Adaptive Immunity," Blood, Vol. 101, No. 9, 2003, pp. 3520-3526. doi:10.1182/blood-2002-10-3063

[9] L. D. Devanur and J. R. Kerr, "Chronic Fatigue Syndrome," Journal of Clinical Virology, Vol. 37, No. 3, 2006, pp. 139-150. doi:10.1016/j.jcv.2006.08.013

[10] S. B. Harvey, M. Wadsworth, S. Wessely and M. Hotopf, "Etiology of Chronic Fatigue Syndrome: Testing Popular Hypotheses Using a National Birth Cohort Study," Psychosomatic Medicine, Vol. 70, No. 4, 2008, pp. 488-495. doi:10.1097/PSY.0b013e31816a8dbc 
[11] D. V. Ablashi, H. B. Eastman, C. B. Owen, M. M. Roman, J. Friedman, J. B. Zabriskie, D. L. Peterson, G. R. Pearson and J. E. Whitman, "Frequent HHV-6 Reactivation in Multiple Sclerosis (MS) and Chronic Fatigue Syndrome (CFS) Patients," Journal of Clinical Virology, Vol. 16, No. 3, 2000, pp. 179-191. doi:10.1016/S1386-6532(99)00079-7

[12] S. H. Beqaj, A. M. Lerner and J. T. Fitzgerald, "Immunoassay with Cytomegalovirus Early Antigens from Gene Products p52 and $\mathrm{CM}_{2}$ (UL44 and UL57) Detects Active Infection in Patients with Chronic Fatigue Syndrome," Journal of Clinical Pathology, Vol. 61, No. 5, 2008, pp. 623-626. doi:10.1136/jep.2007.050633

[13] D. Buchwald, M. H. Wener, T. Pearlman and P. Kith, "Markers of Inflammation and Immune Activation in Chronic Fatigue and Chronic Fatigue Syndrome," Journal of Rheumatology, Vol. 24, No. 2, 1997, pp. 372-376.

[14] E. DeFreitas, B. Hilliard, P. R. Cheney, D. S. Bell, E. Kiggundu, D. Sankey, Z. Wroblewska, M. Palladino, J. P. Woodward and H. Koprowski, "Retroviral Sequences Related to Human T-Lymphotropic Virus Type II in Patients with Chronic Fatigue Immune Dysfunction Syndrome," Proceedings of National Academy of Sciences of the United States of America, Vol. 88, No. 7, 1991, pp. 2922-2926. doi:10.1073/pnas.88.7.2922

[15] D. Gold, R. Bowden, J. Sixbey, R. Riggs, W. J. Katon, R. Ashley, R. M. Obrigewitch and L. Corey, "Chronic Fatigue. A Prospective Clinical and Virologic Study," Journal of the American Medical Association, Vol. 264, 1990, pp. 48-53. doi:10.1001/jama.264.1.48

[16] B. Grinde, "Is Chronic Fatigue Syndrome Caused by a Rare Brain Infection of a Common, Normally Benign Virus?" Medical Hypotheses, Vol. 71, No. 2, 2008, pp. 270-274. doi:10.1016/j.mehy.2008.03.014

[17] J. F. Jones, "Epstein-Barr Virus and the Chronic Fatigue Syndrome: A Short Review," Microbiological Sciences, Vol. 5, No. 12, 1988, pp. 366-369.

[18] A. Linde, B. Andersson, S. B. Svenson, H. Ahrne, M. Carlsson, P. Forsberg, H. Hugo, A. Karstorp, R. Lenkei, A. Lindwall, et al., "Serum Levels of Lymphokines and Soluble Cellular Receptors in Primary Epstein-Barr Virus Infection and in Patients with Chronic Fatigue Syndrome," Journal of Infectious Diseases, Vol. 165, No. 6, 1992, pp. 994-1000. doi:10.1093/infdis/165.6.994

[19] E. W. Brenu, D. R. Staines, O. K. Baskurt, K. J. Ashton, S. B. Ramos, R. M. Christy and S. M. Marshall-Gradisnik, "Immune and Hemorheological Changes in Chronic Fatigue Syndrome," Journal of Translational Medicine, Vol. 8, 2010, p. 1. doi:10.1186/1479-5876-8-1

[20] N. G. Klimas and A. O. Koneru, "Chronic Fatigue Syndrome: Inflammation, Immune Function, and Neuroendocrine Interactions," Current Rheumatology Reports, Vol. 9, No. 6, 2007, pp. 482-487. doi:10.1007/s11926-007-0078-y

[21] M. Maes, I. Mihaylova and J. C. Leunis, "Chronic Fatigue Syndrome Is Accompanied by an IgM-Related Immune Response Directed against Neopitopes Formed by Oxidative or Nitrosative Damage to Lipids and Proteins,"
Neuroendocrinology Letters, Vol. 27, 2006, pp. 615-621.

[22] Racciatti, M. Dalessandro, L. Delle Donne, K. Falasca, P. Zingariello, R. Paganelli, E. Pizzigallo, and J. Vec-chiet, "Study of Immune Alterations in Patients with Chronic Fatigue Syndrome with Different Etiologies," International Journal of Immunopathology and Pharmacology, Vol. 17, No. 2, 2004, pp. 57-62.

[23] F. Friedberg, L. Dechene, M. J. McKenzie II and R. Fontanetta, "Symptom Patterns in Long-Duration Chronic Fatigue Syndrome," Journal of Psychosomatic Research, Vol. 48, No. 1, 2000, pp. 59-68. doi:10.1016/S0022-3999(99)00077-X

[24] "Report of the Working Group on the Possible Relationship between Hepatitis B Vaccination and the Chronic Fatigue Syndrome," Canadian Medical Association Journal, Vol. 149, 1993, pp. 314-319.

[25] S. Appel, J. Chapman and Y. Shoenfeld, "Infection and Vaccination in Chronic Fatigue Syndrome: Myth or Reality?" Autoimmunity, Vol. 40, No. 1, 2007, pp. 48-53. doi: $10.1080 / 08916930701197273$

[26] G. Delage, I. Salit, R. Pennie, M. Alary, B. Duval and B. Ward, "The Possible Relation between Hepatitis B Vaccination and Chronic Fatigue Syndrome," Union Medical Canada, Vol. 122, 1993, pp. 278-279.

[27] D. Ortega-Hernandez and Y. Shoenfeld, "Infection, Vaccination, and Autoantibodies in Chronic Fatigue Syndrome, Cause or Coincidence?" Annals of the New York Academy Sciences, Vol. 1173, 2009, pp. 600-609. doi:10.1111/j.1749-6632.2009.04799.x

[28] K. M. Sleigh, D. G. Danforth, R. T. Hall, J. A. Fleming and H. G. Stiver, "Double-Blind, Randomized Study of the Effects of Influenza Vaccination on the Specific Antibody Response and Clinical Course of Patients with Chronic Fatigue Syndrome," Canadian Journal of Infectious Diseases, Vol. 11, No. 5, 2000, pp. 267-273.

[29] K. M. Sleigh, F. H. Marra and H. G. Stiver, "Influenza Vaccination: Is It Appropriate in Chronic Fatigue Syndrome?" American Journal of Respiratory Medicine, Vol. 1, 2002, pp. 3-9. doi:10.1007/BF03257157

[30] W. Brenu, M. L. van Driel, D. R. Staines, K. J. Ashton, S. B. Ramos, J. Keane, N. G. Klimas and S. M. MarshallGradisnik, "Immunological Abnormalities as Potential Biomarkers in Chronic Fatigue Syndrome/Myalgic Encephalomyelitis," Journal of Translational Medicine, Vol. 9, 2011, p. 81. doi:10.1186/1479-5876-9-81

[31] K. Fukuda, S. E. Straus, I. Hickie, M. C. Sharpe, J. G. Dobbins and A. Komaroff, "The Chronic Fatigue Syndrome: A Comprehensive Approach to Its Definition and Study. International Chronic Fatigue Syndrome Study Group," Annals of Internal Medicine, Vol. 121, No. 12, 1994, pp. 953-959.

[32] "3.9 Influenza," The Australian Immunisation Handbook, 9th Edition, 2012.

http://www.health.gov.au/internet/immunise/publishing.n sf/Content/Handbook-influenza

[33] J. P. Aubry, A. Blaecke, S. Lecoanet-Henchoz, P. Jeannin, N. Herbault, G. Caron, V. Moine and J. Y. Bonnefoy, "Annexin V Used for Measuring Apoptosis in the Early 
Events of Cellular Cytotoxicity," Cytometry, Vol. 37, No. 3, 1991, pp. 197-204. doi:10.1002/(SICI)1097-0320(19991101)37:3<197::AIDCYTO6>3.0.CO;2-L

[34] L. Dickson and K. Finlayson, "VPAC and PAC Receptors: From Ligands to Function," Pharmacology \& Therapeutics, Vol. 121, No. 3, 2009, pp. 294-316. doi:10.1016/i.pharmthera.2008.11.006

[35] P. C. Doherty and A. Kelso, "Toward a Broadly Protective Influenza Vaccine," The Journal of Clinical Investigation, Vol. 118, 2008, pp. 3273-3275.

[36] Y. Ghendon, S. Markushin, Y. Vasiliev, I. Akopova, I. Koptiaeva, G. Krivtsov, O. Borisova, N. Ahmatova, E. Kurbatova, S. Mazurina and V. Gervazieva, "Evaluation of Properties of Chitosan as an Adjuvant for Inactivated Influenza Vaccines Administered Parenterally," Journal of Medical Virology, Vol. 81, No. 3, 2009, pp. 494-506. doi:10.1002/jmv.21415

[37] S. M. Hsieh and S. C. Chang, "Insufficient Perforin Expression in $\mathrm{CD}^{+} \mathrm{T}$ Cells in Response to Hemagglutinin from Avian Influenza (H5N1) Virus," Journal of Immunology, Vol. 176, No. 6, 2006, pp. 4530-4533.

[38] X. S. He, T. H. Holmes, K. Mahmood, G. W. Kemble, C. L. Dekker, A. M. Arvin and H. B. Greenberg, "Phenotypic Changes in Influenza-Specific $\mathrm{CD}^{+} \mathrm{T}$ Cells after Immunization of Children and Adults with Influenza Vaccines," The Journal of Infectious Diseases, Vol. 197, No. 6, 2008, pp. 803-811. doi:10.1086/528804

[39] J. Mysliwska, P. Trzonkowski, E. Szmit, L. B. Brydak, M. Machala and A. Mysliwski, "Immunomodulating Effect of Influenza Vaccination in the Elderly Differing in Health Status," Experimental Gerontology, Vol. 39, No. 10, 2004, pp. 1447-58. doi:10.1016/j.exger.2004.08.005

[40] J. E. McElhaney, S. Gravenstein, C. M. Upshaw, J. W. Hooton, P. Krause, P. Drinka and R. C. Bleackley,
"Granzyme B: A Marker of Risk for Influenza in Institutionalized Older Adults," Vaccine, Vol. 19, No. 27, 2001, pp. 3744-3751. doi:10.1016/S0264-410X(01)00087-1

[41] J. M. Schapiro, Y. Segev, L. Rannon, M. Alkan and B. Rager-Zisman, "Natural Killer (NK) Cell Response after Vaccination of Volunteers with Killed Influenza Vaccine," Journal of Medical Virology, Vol. 30, No. 3, 1990, pp. 196-200. doi:10.1002/jmv. 1890300310

[42] R. Clark, N. B. Anderson, V. R. Clark and D. R. Williams, "Racism as a Stressor for African Americans. A Biopsychosocial Model," The American Psychologist, Vol. 54, No. 10, 1999, pp. 805-816. doi:10.1037/0003-066X.54.10.805

[43] V. Demicheli, D. Rivetti, J. J. Deeks and T. O. Jefferson, "Vaccines for Preventing Influenza in Healthy Adults," Cochrane Database of Systematic Reviews, 2000, Article ID: CD001269.

[44] I. Rosenkrands, C. Vingsbo-Lundberg, T. J. Bundgaard, T. Lindenstrom, V. Enouf, S. van der Werf, P. Andersen and E. M. Agger, "Enhanced Humoral and Cell-Mediated Immune Responses after Immunization with Trivalent Influenza Vaccine Adjuvanted with Cationic Liposomes," Vaccine, Vol. 29, No. 37, 2011, pp. 6283-6291. doi:10.1016/j.vaccine.2011.06.040

[45] J. E. McElhaney, S. Gravenstein, P. Krause, J. W. Hooton, C. M. Upshaw and P. Drinka, "Assessment of Markers of the Cell-Mediated Immune Response after Influenza Virus Infection in Frail Older Adults," Clinical and Vaccine Immunology, Vol. 5, No. 6, 1998, pp. 840-844.

[46] J. E. McElhaney, S. Gravenstein, C. M. Upshaw, J. W. Hooton, P. Krause and P. Drinka, "Immune Response to Influenza Vaccination in Institutionalized Elderly: Effect on Different T-Cell Subsets," Vaccine, Vol. 16, No. 4, 1998, pp. 403-409. doi:10.1016/S0264-410X(97)80918-8 